\title{
Spectral Restoration Based Speech Enhancement for Robust Speaker Identification
}

\author{
Nasir Saleem*1, Tayyaba Gul Tareen² \\ ${ }^{1}$ Department of Electrical Engineering, Gomal University, D.I.Khan (Pakistan) \\ ${ }^{2}$ Department of Electrical Engineering, Iqra University, Peshawar (Pakistan)
}

Received 8 October 2017 | Accepted 21 December 2017 | Published 19 January 2018

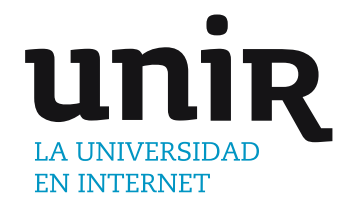

\section{ABSTRACT}

Spectral restoration based speech enhancement algorithms are used to enhance quality of noise masked speech for robust speaker identification. In presence of background noise, the performance of speaker identification systems can be severely deteriorated. The present study employed and evaluated the Minimum Mean-SquareError Short-Time Spectral Amplitude Estimators with modified a priori SNR estimate prior to speaker identification to improve performance of the speaker identification systems in presence of background noise. For speaker identification, Mel Frequency Cepstral coefficient and Vector Quantization is used to extract the speech features and to model the extracted features respectively. The experimental results showed significant improvement in speaker identification rates when spectral restoration based speech enhancement algorithms are used as a pre-processing step. The identification rates are found to be higher after employing the speech enhancement algorithms.

\section{KEYWORDS}

\author{
A Priori SNR, Spectral \\ Restoration, Speech \\ Enhancement, Speaker \\ Identification, Mel \\ Frequency Cepstral \\ Coefficients, Vector \\ Quantization.
}

OI: $10.9781 /$ ijimai.2018.01.002

\section{INTRODUCTION}

\begin{abstract}
C PEECH enhancement aspires to improve quality by employing $\checkmark$ a variety of speech processing algorithms. The intention of the enhancement is to improve the speech intelligibility and/or overall perceptual quality of speech noise masked speech. Enhancement of speech degraded by background noise, called noise reduction is a significant area of speech enhancement and is considered for diverse applications for example, mobile phones, speech/speaker recognition/ identification [1] and hearing aids. The speech signals are frequently contaminated by the background noise, which affects the performance of speaker identification (SID) systems. The SID systems are used in online banking, voice mail, remote computer access etc. Therefore, for effective use of such systems, a speech enhancement system must be positioned in front-end to improve identification accuracy. Fig.1 shows the procedural block diagram of speech enhancement and speaker identification system. The algorithms for speech enhancement are categorized into three fundamental classes, (i) filtering techniques including spectral subtraction [2-5] Wiener filtering [6-8] and signal subspace techniques [9-10], (ii) Spectral restoration algorithms including Mean-Square-Error Short-Time Spectral Amplitude Estimators [11-12] and (iii) speech-model based algorithms. The systems presented in [6-8, 11-13] principally depend on accurate estimates of signal-to-noise ratio (SNR) in all frequency bands, because gain is computed as function of spectral SNR. A conventional and recognized technique for SNR estimation is decision-directed (DD) method suggested in [11] The DD technique tails the shape of instantaneous SNR for a priori SNR
\end{abstract}

\footnotetext{
* Corresponding author.

E-mail address: nasirsaleem@gu.edu.pk
}

estimate and brings one-frame delay. Therefore, to avoid one-frame delay, momentum terms are incorporated to get better tracking speed of system and avoid the frame delay problem. All the mentioned systems in [11-13] can significantly improve speech quality. Binary masking [14-18] is another class that increases speech quality and intelligibility simultaneously. This paper presents Mean-Square-Error Short-Time Spectral Amplitude Estimators with modified a priori SNR estimation to reduce background noise and to improve identification rates of speaker identification systems in presence of background noises. The paper is prepared as follows. Section 2 presents the overview of speech enhancement system; section 3 gives speaker identification system; section 4 presents the experimental setup, results and discussions, and section 5 presents the summary and concluding remarks. The Matlab $\mathrm{R} 2015 \mathrm{~b}$ is used to construct the algorithms and simulations.

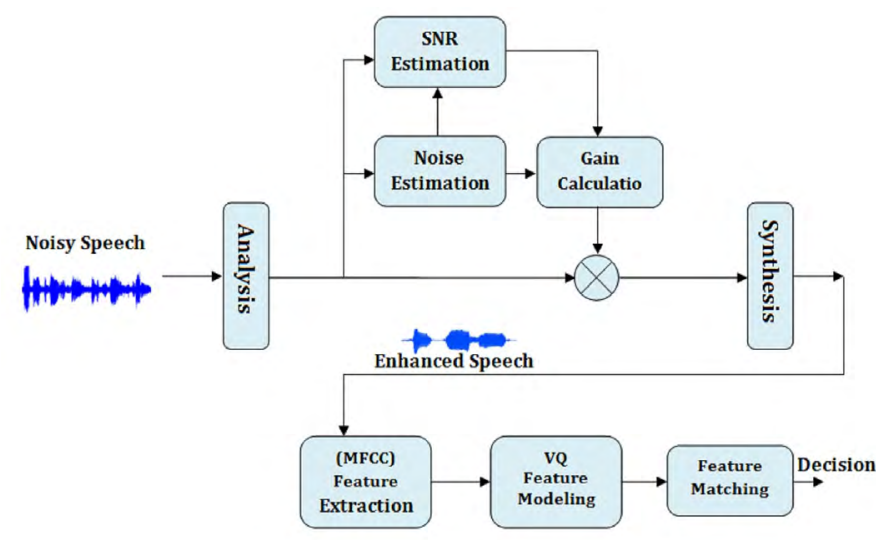

Fig. 1. Procedural block diagram of Speech enhancement and speaker identification system. 


\section{Spectral Restoration Based Speech Enhancement System}

In classical spectral restoration based speech enhancement system, the noisy speech is given as; $\mathrm{y}(\mathrm{t})=\mathrm{s}(\mathrm{t})+\mathrm{n}(\mathrm{t})$, where $\mathrm{s}(\mathrm{t})$ and $\mathrm{n}(\mathrm{t})$ specify clean speech and noise signal respectively. Let $\mathrm{Y}(\mathrm{k}, \omega \mathrm{k}), \mathrm{S}(\mathrm{k}, \omega \mathrm{k})$ and $\mathrm{N}(\mathrm{k}, \omega \mathrm{k})$ show $\mathrm{y}(\mathrm{t}), \mathrm{s}(\mathrm{t})$ and $\mathrm{n}(\mathrm{t})$ respectively with spectral element $\omega k$ and time frame $k$. The quasi-stationary nature of speech is considered in frame analysis since noise and speech signals both reveal non-stationary behavior. A speech enhancement algorithm involves in multiplication of a spectral gain $G(k, \omega k)$ to short-time spectrum $Y(k, \omega k)$ and the computation of spectral gain follows two key parameters, a posteriori SNR and the $a$ priori SNR estimation:

$$
\begin{gathered}
\gamma\left(\mathrm{k}, \omega_{\mathrm{k}}\right)=\frac{\left|\mathrm{Y}\left(\mathrm{k}, \omega_{\mathrm{k}}\right)\right|^{2}}{\mathrm{E}\left\{\left|\mathrm{N}\left(\mathrm{k}, \omega_{\mathrm{k}}\right)\right|^{2}\right\}}=\frac{\left|\mathrm{Y}\left(\mathrm{k}, \omega_{\mathrm{k}}\right)\right|^{2}}{\sigma_{\mathrm{n}}^{2}\left(\mathrm{k}, \omega_{\mathrm{k}}\right)} \\
\xi\left(\mathrm{k}, \omega_{\mathrm{k}}\right)=\frac{\mathrm{E}\left\{\left|\mathrm{S}\left(\mathrm{k}, \omega_{\mathrm{k}}\right)\right|^{2}\right\}}{\mathrm{E}\left\{\left|\mathrm{N}\left(\mathrm{k}, \omega_{\mathrm{k}}\right)\right|^{2}\right\}}=\frac{\sigma_{\mathrm{s}}^{2}\left(\mathrm{k}, \omega_{\mathrm{k}}\right)}{\sigma_{\mathrm{n}}^{2}\left(\mathrm{k}, \omega_{\mathrm{k}}\right)}
\end{gathered}
$$

Where $\mathrm{E}\{$.$\} shows expectation operator, \gamma\left(\mathrm{k}, \omega_{\mathrm{k}}\right)$ and $\xi\left(\mathrm{k}, \omega_{\mathrm{k}}\right)$ presents a posteriori SNR estimation and a priori SNR estimation. In practical implementations of a speech enhancement system, squared power spectrum density of clean speech $\left|\mathrm{X}\left(\mathrm{k}, \omega_{\mathrm{k}}\right)\right|^{2}$ and noise $\left|\mathrm{D}\left(\mathrm{k}, \omega_{\mathrm{k}}\right)\right|^{2}$ are unrevealed as only noisy speech is available. Therefore; both instantaneous and a priori SNR need to be estimated. The noise power spectral density is estimated during speech gaps exploiting standard recursive relation, given as:

$$
\hat{\sigma}_{n}^{2}\left(k, \omega_{k}\right)=\beta \hat{\sigma}_{n}^{2}\left(k-1, \omega_{k}\right)+(1-\beta) \dot{\sigma}_{Y}^{2}\left(k-1, \omega_{k}\right)
$$

Where, $\beta$ is the smoothing factor and $\tilde{\sigma}_{Y}^{2}\left(k-1, \omega_{k}\right)$ is estimation in previous frame. The SNR can be calculated as:

$$
\begin{aligned}
& \operatorname{SNR}_{\text {INST }}\left(\mathrm{k}, \omega_{\mathrm{k}}\right)=\frac{\left|\mathrm{S}\left(\mathrm{k}, \omega_{\mathrm{k}}\right)\right|^{2}}{\left|\mathrm{~N}\left(\mathrm{k}, \omega_{\mathrm{k}}\right)\right|^{2}} \\
& \xi_{\mathrm{DD}}\left(\mathrm{k}, \omega_{\mathrm{k}}\right)=\alpha \frac{\left|\mathrm{G}\left(\mathrm{k}-1, \omega_{\mathrm{k}}\right) * \mathrm{Y}\left(\mathrm{k}, \omega_{\mathrm{k}}\right)\right|^{2}}{\hat{\sigma}_{\mathrm{n}}^{2}\left(\mathrm{k}, \omega_{\mathrm{k}}-1\right)} \\
& +(1-\alpha) \mathrm{F}\left\{\gamma\left(\mathrm{k}, \omega_{\mathrm{k}}\right)-1\right\}
\end{aligned}
$$

Where $\alpha$ is smoothing factor and has a constant value $0.98, \xi_{\mathrm{DD}}\left(\mathrm{k}, \omega_{\mathrm{k}}\right)$ is a priori noise estimate via decision-direct (DD) method whereas $\mathrm{F}\{$.$\} is half-wave rectification. By setting \alpha$ as a fixed value near to 1 , the DD approach introduces less residual noise. However, it may lead to delay in estimation since a fixed value cannot track the rapid change of speech. The DD is an efficient method and achieves well in speech enhancement applications however; the a priori SNR follows the shape of instantaneous SNR and brings single-frame delay. To overcome single-frame delay, a modified form of DD method is used to estimate a priori $\mathrm{SNR}$. The modified a priori $\mathrm{SNR}$ is written as:

$$
\begin{aligned}
& \xi_{\mathrm{MDD}}\left(\mathrm{k}, \omega_{\mathrm{k}}\right)=\alpha \frac{\left|\mathrm{G}\left(\mathrm{k}-1, \omega_{\mathrm{k}}\right)^{*} \mathrm{Y}\left(\mathrm{k}, \omega_{\mathrm{k}}\right)\right|^{2}}{\hat{\sigma}_{\mathrm{n}}^{2}\left(\mathrm{k}, \omega_{\mathrm{k}}-1\right)} \\
& +\mu\left(\mathrm{k}, \omega_{\mathrm{k}}\right)+(1-\alpha) \mathrm{F}\left\{\gamma\left(\mathrm{k}, \omega_{\mathrm{k}}\right)-1\right\}
\end{aligned}
$$

$$
\mu\left(\mathrm{k}, \omega_{\mathrm{k}}\right)=\zeta\left[\xi_{\text {PRIO }}\left(\mathrm{k}-1, \omega_{\mathrm{k}}\right)-\xi_{\text {PRIO }}\left(\mathrm{k}-2, \omega_{\mathrm{k}}\right)\right]
$$

Equation (6) shows the modified DD (MDD) version used in the speech enhancement system, $\alpha$ is smoothing parameter $(\alpha=0.98), \zeta$ is momentum parameter $(\zeta=0.998), \mu\left(\mathrm{m}, \omega_{\mathrm{k}}\right)$ shows momentum terms and $\lambda_{\mathrm{D}}\left(\mathrm{m}, \omega_{\mathrm{k}}\right)$ is the estimation of background noise variance. The $\xi_{\mathrm{MDD}}\left(\mathrm{k}, \omega_{\mathrm{k}}\right)$ shows a priori SNR estimation after modification. The estimated power spectrum of the clean speech magnitude $\mathrm{S}_{\mathrm{EST}}\left(\mathrm{k}, \omega_{\mathrm{k}}\right)$ is attained by multiplying gain function with noisy speech $\mathrm{Y}\left(\mathrm{k}, \omega_{\mathrm{k}}\right)$ as:

$$
\left|\mathrm{S}_{\mathrm{EST}}\left(\mathrm{k}, \omega_{\mathrm{k}}\right)\right|=\left|\mathrm{Y}\left(\mathrm{k}, \omega_{\mathrm{k}}\right)\right| * \mathrm{G}\left(\mathrm{k}, \omega_{\mathrm{k}}\right)
$$

The gain function $\mathrm{G}\left(\mathrm{k}, \omega_{\mathrm{k}}\right)$ is given as:

$$
\begin{aligned}
& \mathrm{G}\left(\mathrm{k}, \omega_{\mathrm{k}}\right)=\min \left\{\zeta, \frac{\xi\left(\mathrm{k}, \omega_{\mathrm{k}}\right)}{1+\xi\left(\mathrm{k}, \omega_{\mathrm{k}}\right)}\left[\frac{1}{2} \int_{\mathrm{v}\left(\mathrm{k}, \omega_{\mathrm{k}}\right)}^{\infty} \frac{\mathrm{e}^{-\mathrm{t}} \mathrm{t}}{\mathrm{t}}\right]\right\} \\
& \mathrm{v}\left(\mathrm{k}, \omega_{\mathrm{k}}\right)=\frac{\xi\left(\mathrm{k}, \omega_{\mathrm{k}}\right)}{1+\xi\left(\mathrm{k}, \omega_{\mathrm{k}}\right)} \gamma\left(\mathrm{k}, \omega_{\mathrm{k}}\right)
\end{aligned}
$$

Where, $\varsigma$ is used to avoid large gain values at low a posteriori SNR and $\varsigma=10$ is chosen here.

\section{SPEAKER IDENTIFICATION SySTEM}

The intention of a Speaker identification system is to identity information regarding any speaker which is categorized into two sub-categories called as Speaker identification (SID) and speaker Verification (SVR). For SID, the Mel Frequency Cepstral coefficient (MFCC) and Vector Quantization (VQ) is used to extract the speech features and to model the extracted features respectively. The speaker identification system drives in two stages, the training and testing stages. In training mode the system is allowed to create the database of speech signals and formulate a feature model of speech utterances. In testing mode, the system uses information provided in database and attempts to segregate and identify the speakers. Here, the Mel frequency Cepstral Coefficients (MFCCs) features are used for constructing a SID system. The extracted features of speakers are quantized to a number of centroids employing vector quantization (VQ) K-means algorithm. MFCCs are computed in training as well as in testing stage. The Euclidean distance among MFCCs of all speakers in training stage to centroids of isolated speaker in testing stage is calculated and a particular speaker is identified according to minimum Euclidean distance.

\section{A. Feature Extraction}

The MFCCs are acquired by pre-emphasis of speech initially to emphasize high frequencies and eliminate glottal and lip radiations. The resulting speech is fragmented, windowed, and FFT is computed to attain spectra. To estimate human auditory system, triangular bandpass filters bank is utilized. A linear scale is used to compute center frequencies which are lower than $1 \mathrm{kHz}$, while logarithmic scale is considered for center frequencies higher than $1 \mathrm{kHz}$. The filter bank response is given in Fig. 2. The Mel-spaced filter bank response is given as:

$$
\operatorname{Mel}(f)=2595 \log \left(1+\frac{f}{700}\right)
$$

The DFT is computed on log of Mel spectrum to figure Cepstrum as:

$$
\mathrm{M}_{\mathrm{k}}=\sqrt{\frac{2}{\mathrm{~N}_{\mathrm{f}}}} \sum_{\mathrm{n}=1}^{\mathrm{N}_{\mathrm{f}}} \log (\dot{\mathrm{S}}(\mathrm{n})) \cos \left(\frac{\mathrm{g} \pi}{\mathrm{N}_{\mathrm{f}}}(\mathrm{n}-0.5)\right)
$$

Where Mg shows MFCCs, $\dot{\mathrm{S}}$ is $\mathrm{n}^{\text {th }}$ Mel filter output, $K$ is number of 
MFCCs chosen between 5 to 26 , and $\mathrm{N} f$ is the number of Mel filters. Initially few coefficients are considered since most of the specific information about speakers is present in them.

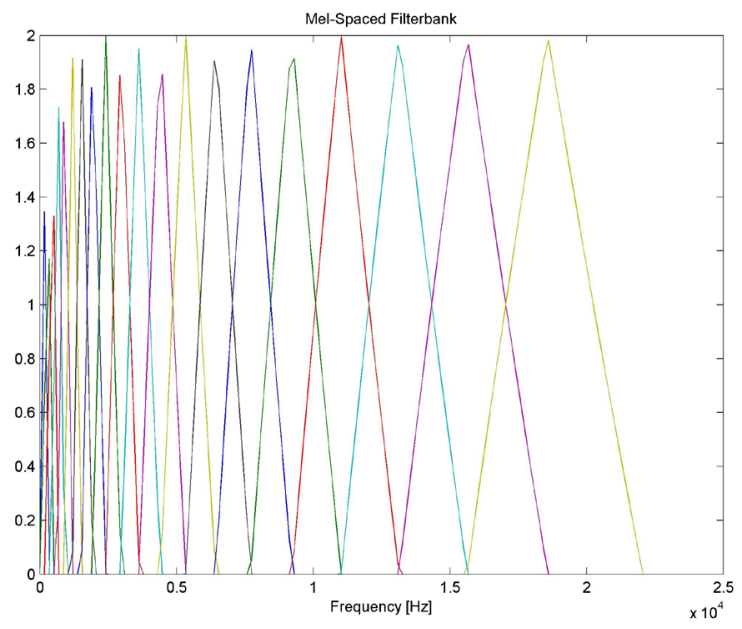

Fig. 2. Mel-Spaced Filter bank Response.

\section{B. Vector Quantization}

Vector quantization (VQ) is a lossy compression method based on the block coding theory [20]. The purpose of VQ in speaker recognition systems is to create a classification system for every speaker and a large set of acoustic vectors are converted to lesser set that signifies centroids of distribution shown in Fig. 3. The VQ is employed since all MFCC generated feature vector cannot be stored and extracted acoustic vectors are clustered into a set of codewords (referred to as codebook) and this clustering is achieved by using the K-Means Algorithm which separates the $\mathrm{M}$ feature vectors into $\mathrm{K}$ centroids. Initially $\mathrm{K}$ clustercentroids are chosen randomly within $\mathrm{M}$ feature vectors and then all feature vectors are allocated to nearby centroid, and the creating the centroids, all other new clusters follow the same pattern. The process keeps on until a certain condition for stopping is reached, i.e., the mean square error (MSE) among acoustic vector and cluster centroid is lower than a certain predefined threshold or there are no additional variations in cluster-center task [21].
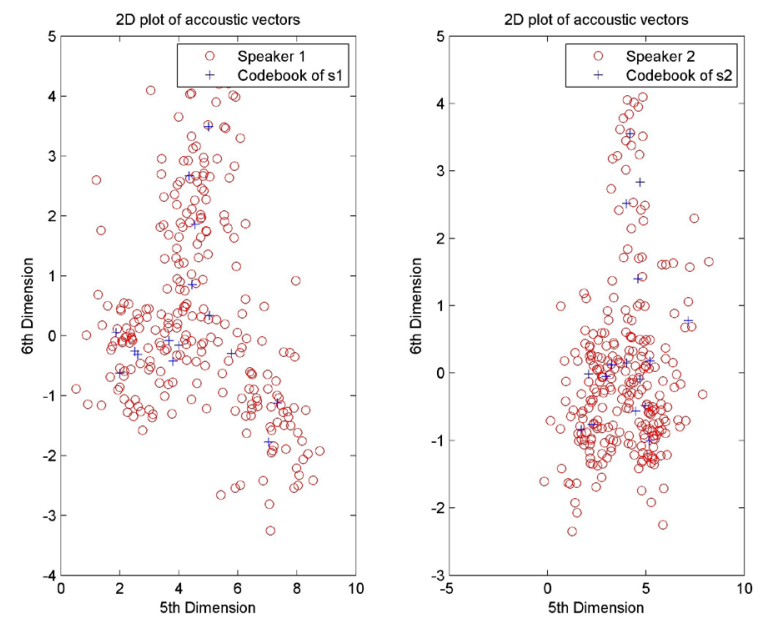

Fig. 3. 2D acoustic Vector analysis for speakers.

\section{Speaker Identification}

The speaker recognition phase is characterized by a set of acoustic feature vectors $\{M 1, M 2, \ldots ., M t\}$, and is judged against codebooks in list. For all codebooks a distortion is calculated, and a speaker with the lowest distortion is selected, and this distortion is the sum of squared Euclidean distances among vectors and their centroids. As a result, all feature vectors in $\mathrm{M}$ sequence are compared with codebooks, and the codebooks with the minimum average distance are selected. The Euclidean distance between two points, $\lambda=\left(\lambda_{1}, \lambda_{2} \ldots \lambda_{n}\right)$ and $\eta=\left(\eta_{1}, \eta_{2} \ldots\right.$ $\left.\eta_{n}\right)$ is given by [21-22]:

$$
\begin{aligned}
& \sqrt{\left[(\lambda-\gamma)^{2}+(\lambda-\gamma)^{2}+(\lambda-\gamma)^{2}+\ldots+(\lambda-\gamma)^{2}\right]}= \\
& \sqrt{\sum_{i=1}^{n}(\lambda-\gamma)^{2}}
\end{aligned}
$$

\section{RESUlts AND Discussion}

Six different speakers, three male and three female, were selected from Noizeus [23] and TIMIT database, respectively, while 50 speech sentences uttered by the speakers are considered during training stage for speaker identification. In testing stage, speech utterances are selected at random to access the identification rates. To evaluate performance of system, four signal-to-noise ratio levels, including $0 \mathrm{~dB}, 5 \mathrm{~dB}, 10 \mathrm{~dB}$ and $15 \mathrm{~dB}$ are used. Also three noisy situations including car, street and white noise are used to degrade the clean speech. The Perceptual evaluation of speech quality (PESQ) [23] and Segmental SNR (SNRSeg) [24] is used to predict the speech quality after speech enhancement. Three sets of experiments are conducted to measure the speaker identification rates including, clean speech with no background noise, speech degraded by background noise and speech processed by the spectral restoration enhancing algorithms. The presented system is compared to various baseline state-of-art speech enhancement algorithms. The baseline algorithms include MMSE, Spectral subtraction (SS), and signal subspace (Sig_Sp). Table I shows the PESQ scores obtained with the spectral restoration based algorithm and baseline algorithms. The proposed algorithm performed very well in noisy environments and at all SNR levels against baseline speech enhancement algorithms. A considerable improvement in PESQ scores is evident which shows that the proposed speech enhancement algorithm effectively reduced various background noise sources from target speech. Similarly, Fig. 4 shows PESQ scores obtained after applying Minimum Mean-SquareError Short-Time Spectral Amplitude Estimators with modified $a$ priori SNR estimate (MMSE-MDD). The modified version offers the best results consistently in all SNR levels and noisy conditions when compared to noisy and speech processed by traditional MMSESTSA speech enhancement algorithm. Table II shows the SNRSeg results obtained with the spectral restoration based algorithm and baseline algorithms. Again in terms of SNRSeg, the proposed speech enhancement algorithm outperformed against baseline algorithms. Significant SNRSeg improvements are evident from the obtained results. Fig. 5 shows the speech quality in terms of segmental SNR (SNRSeg) where highest SNRSeg scores are obtained with MMSEMDD. The enhanced speech associated with six speakers is tested for speaker identification. Table III offers the percentage identification rates achieved with proposed speech enhancement algorithm against baseline algorithms. The speaker identification rates are remarkably improved with the proposed algorithm in various noise environments at all SNR levels as compared to baseline algorithms and unprocessed noisy speech. At low SNR (0dB) a significant increase in identification rates is observed in all noise environments which clearly showed that the noise is effectively eliminated. Fig. 6 shows the identification rates, the lowest identification rates are observed in presence of background noise (Babble, car and street) however, employment of the speech enhancement before speaker identification has tremendously increased the identification rates which are evident in Fig.5. The identification rates for MMSE-MDD are higher in all SNR conditions and levels. 
TABLE I. PESQ Analysis Against Baseline Speech Enhancement Algorithms and Noisy Speech

\begin{tabular}{|c|c|c|c|c|c|c|}
\hline Noise Type & $\begin{array}{c}\text { SNR } \\
\text { (in dB) }\end{array}$ & $\begin{array}{c}\text { Noisy } \\
\text { Speech }\end{array}$ & $\begin{array}{c}\text { Spectral } \\
\text { Subtraction }\end{array}$ & Signal Subspace & MMSE & Proposed \\
\hline \multirow{4}{*}{ Babble Noise } & 0 & 1.72 & 1.89 & 1.91 & 1.89 & 1.97 \\
\hline & 5 & 2.11 & 2.19 & 2.29 & 2.23 & 2.35 \\
\hline & 10 & 2.43 & 2.53 & 2.61 & 2.55 & 2.69 \\
\hline & 15 & 2.66 & 2.71 & 2.76 & 2.71 & 2.83 \\
\hline \multirow{4}{*}{ Car Noise } & 0 & 1.79 & 1.91 & 2.01 & 1.87 & 2.07 \\
\hline & 5 & 1.97 & 2.23 & 2.31 & 2.21 & 2.45 \\
\hline & 10 & 2.31 & 2.42 & 2.62 & 2.61 & 2.72 \\
\hline & 15 & 2.45 & 2.56 & 2.76 & 2.78 & 2.91 \\
\hline \multirow{4}{*}{ Street Noise } & 0 & 1.77 & 1.93 & 1.96 & 1.88 & 2.13 \\
\hline & 5 & 2.05 & 2.21 & 2.31 & 2.12 & 2.43 \\
\hline & 10 & 2.41 & 2.57 & 2.59 & 2.55 & 2.69 \\
\hline & 15 & 2.54 & 2.65 & 2.69 & 2.61 & 2.86 \\
\hline
\end{tabular}

TABLE II. Segmental SNR (SNRSeg) Analysis Against Baseline Speech Enhancement Algorithms and Noisy Speech

\begin{tabular}{|c|c|c|c|c|c|c|}
\hline Noise Type & $\begin{array}{c}\text { SNR } \\
\text { (in dB) }\end{array}$ & $\begin{array}{c}\text { Noisy } \\
\text { Speech }\end{array}$ & $\begin{array}{c}\text { Spectral } \\
\text { Subtraction }\end{array}$ & Signal Subspace & MMSE & Proposed \\
\hline \multirow{4}{*}{ Babble } & 0 & 0.11 & 1.21 & 1.55 & 1.12 & 1.66 \\
\hline & 5 & 1.13 & 1.77 & 1.89 & 1.83 & 2.01 \\
\hline & 10 & 1.45 & 2.11 & 2.17 & 1.99 & 2.37 \\
\hline & 15 & 1.64 & 2.34 & 2.38 & 2.28 & 2.44 \\
\hline \multirow{4}{*}{ Car } & 0 & 0.10 & 1.32 & 1.28 & 1.13 & 1.63 \\
\hline & 5 & 1.23 & 1.89 & 1.93 & 1.78 & 1.98 \\
\hline & 10 & 1.56 & 2.14 & 2.21 & 1.97 & 2.41 \\
\hline & 15 & 1.66 & 2.29 & 2.33 & 2.37 & 2.57 \\
\hline \multirow{4}{*}{ Street } & 0 & 0.18 & 1.29 & 1.41 & 1.16 & 1.59 \\
\hline & 5 & 1.43 & 1.88 & 1.92 & 1.72 & 1.99 \\
\hline & 10 & 1.53 & 2.21 & 2.23 & 2.01 & 2.39 \\
\hline & 15 & 1.67 & 2.35 & 2.39 & 2.21 & 2.51 \\
\hline
\end{tabular}

TABLE III. Speaker Identification Rates of Speech Enhancement Algorithms (in Percentage)

\begin{tabular}{|c|c|c|c|c|c|c|}
\hline Noise Type & $\begin{array}{c}\text { SNR } \\
\text { (in dB) }\end{array}$ & $\begin{array}{c}\text { Noisy } \\
\text { Speech }\end{array}$ & $\begin{array}{c}\text { Spectral } \\
\text { Subtraction }\end{array}$ & Signal Subspace & MMSE & Proposed \\
\hline \multirow{4}{*}{ Babble } & 0 & 41 & 52 & 55 & 56 & 62 \\
\hline & 5 & 58 & 64 & 67 & 69 & 71 \\
\hline & 10 & 77 & 81 & 83 & 84 & 79 \\
\hline & 15 & 85 & 88 & 89 & 88 & 91 \\
\hline \multirow{4}{*}{ Car } & 0 & 40 & 51 & 53 & 55 & 58 \\
\hline & 5 & 56 & 66 & 69 & 71 & 73 \\
\hline & 10 & 76 & 81 & 85 & 87 & 88 \\
\hline & 15 & 82 & 89 & 89 & 90 & 91 \\
\hline \multirow{4}{*}{ Street } & 0 & 38 & 49 & 54 & 57 & 59 \\
\hline & 5 & 46 & 67 & 69 & 71 & 73 \\
\hline & 10 & 71 & 80 & 82 & 86 & 88 \\
\hline & 15 & 80 & 85 & 87 & 90 & 92 \\
\hline
\end{tabular}

\section{V.. Summary AND CONClusions}

This paper presents the Mean-Square-Error Short-Time Spectral Amplitude Estimators with modified a priori SNR estimation to reduce the background noise and to improve identification rates of speaker identification systems in presence of background noises. The lowest identification rates are reported when background noises such as babble, car and street are present. By implementing the proposed speech enhancement algorithm as pre-processing step, the identification rates are increased about $40 \%, 38 \%$ and $35 \%$ at low SNR level (0dB) in all noise environments. The proposed speech enhancement algorithm offered significant improvements in terms of PESQ and SNRSeg scores. The speaker identification rates are higher than baseline algorithms in all noise environments and at all SNR levels consistently. In presence of noise, it is difficult to identify specific speaker, however; the use of a speech enhancement system prior to speaker identification remarkably increased the identification rates. On the basis of experimental results, it is concluded that the use of the proposed speech enhancement algorithm as preprocessor can remarkably increase the speaker identification in many noisy environments as compared to many other speech enhancement algorithms. 

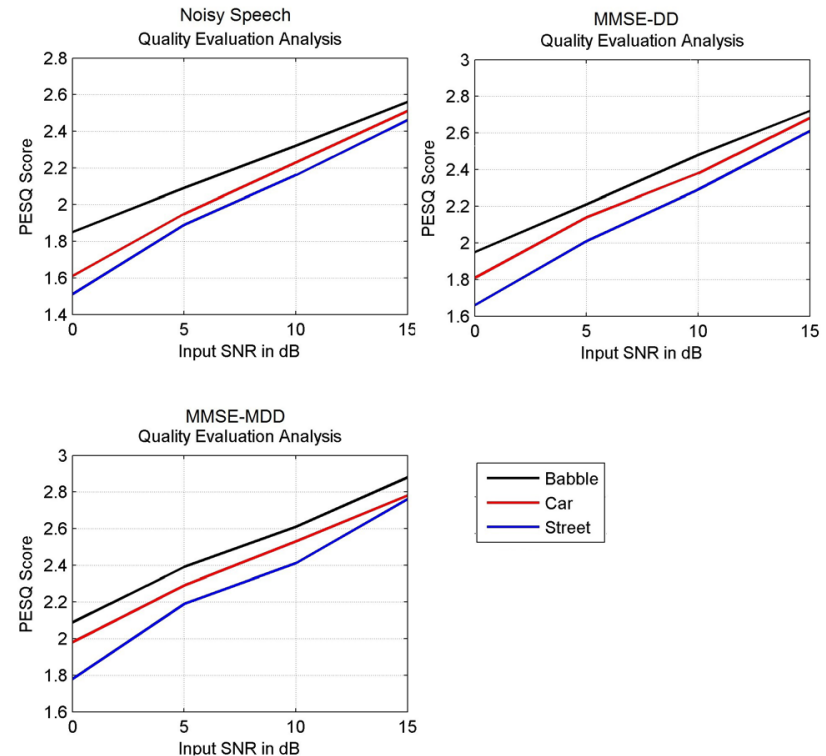

Fig. 4. PESQ Analysis.
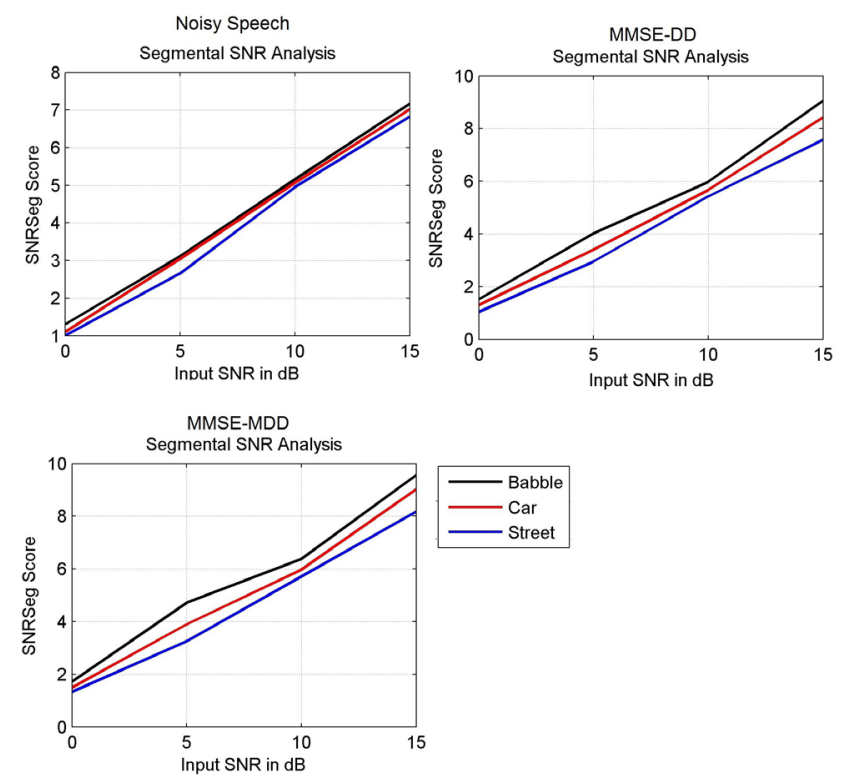

Fig. 5. SNRSeg Analysis.
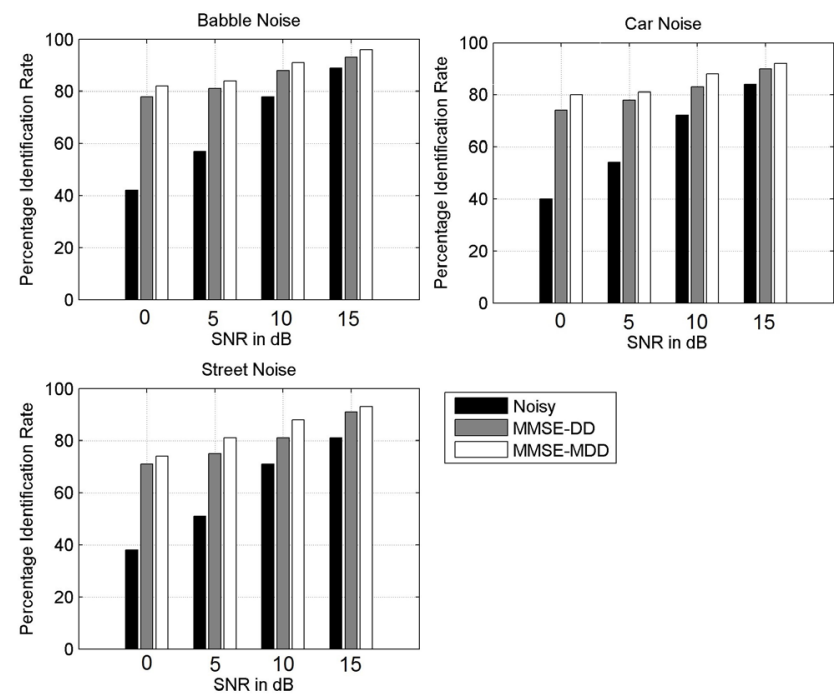

Fig. 6. Speaker identification rate analysis.

\section{REFERENCES}

[1] Hicham, E.M., Akram, H., Khalid, S. (2016) Using features of local densities, statistics and HMM toolkit (HTK) for offline Arabic handwriting text recognition. J. Electr. Syst. Inform. Technol., 4(3), 387-396. http:// dx.doi.org/10.1016/j.jesit.2016.07.005

[2] Berouti, M., Schwartz, M., and Makhoul, J. (1979). Enhancement of speech corrupted by acoustic noise. Proc. IEEE Int. Conf. Acoust., Speech, Signal Processing, pp: 208-211.

[3] Kamath, S. and Loizou, P. (2002). A multi-band spectral subtraction method for enhancing speech corrupted by colored noise. IEEE Int. Conf. Acoust., Speech, Signal Processing, vol. 4, pp. 44164-44164.

[4] Gustafsson, H., Nordholm, S., and Claesson, I. (2001). Spectral subtraction using reduced delay convolution and adaptive averaging. IEEE Trans. on Speech and Audio Processing, 9(8), 799-807.

[5] Saleem N, Ali S, Khan U and Ullah F, (2013). Speech Enhancement with Geometric Advent of Spectral Subtraction using Connected TimeFrequency Regions Noise Estimation. Research Journal of Applied Sciences, Engineering and Technology, 6(06), 1081-1087.

[6] Lim, J. and Oppenheim, A. V. (1978). All-pole modeling of degraded speech. IEEE Trans. Acoust., Speech, Signal Proc., ASSP-26(3), 197210.

[7] Scalart, P. and Filho, J. (1996). Speech enhancement based on a priori signal to noise estimation. Proc. IEEE Int. Conf. Acoust., Speech, Signal Processing, 629-632.

[8] Hu, Y. and Loizou, P. (2004). Speech enhancement based on wavelet thresholding the multitaper spectrum. IEEE Trans. on Speech and Audio Processing, 12(1), 59-67.

[9] Hu, Y. and Loizou, P. (2003). A generalized subspace approach for enhancing speech corrupted by colored noise. IEEE Trans. on Speech and Audio Processing, 11, 334-341.

[10] Jabloun, F. and Champagne, B. (2003). Incorporating the human hearing properties in the signal subspace approach for speech enhancement. IEEE Trans. on Speech and Audio Processing, 11(6), 700-708.

[11] Ephraim, Y. and Malah, D. (1984). Speech enhancement using a minimum mean-square error short-time spectral amplitude estimator. IEEE Trans. Acoust.,Speech, Signal Process., ASSP-32(6), 1109-1121.

[12] Ephraim, Y. and Malah, D. (1985). Speech enhancement using a minimum mean-square error log-spectral amplitude estimator. IEEE Trans. Acoust., Speech, Signal Process., ASSP-23(2), 443-445.

[13] Cohen, I. (2002). Optimal speech enhancement under signal presence uncertainty using log-spectra amplitude estimator. IEEE Signal Processing Letters, 9(4), 113-116.

[14] Saleem, N. (2016), Single channel noise reduction system in low SNR. International Journal of Speech Technology, 20(1), 89-98. doi: 10.1007/ s10772-016-9391-z

[15] Saleem, N., Mustafa, E., Nawaz, A., \& Khan, A. (2015). Ideal binary masking for reducing convolutive noise. International Journal of Speech Technology, 18(4), 547-554. doi:10.1007/s10772-015-9298-0

[16] Saleem, N., Shafi, M., Mustafa, E., \& Nawaz, A. (2015). A novel binary mask estimation based on spectral subtraction gain induced distortions for improved speech intelligibility and quality. Technical Journal, UET, Taxila, 20(4), 35-42.

[17] Boldt, J. B., Kjems, U., Pedersen, M. S., Lunner, T., \& Wang, D. (2008). Estimation of the ideal binary mask using directional systems. In Proc. Int. Workshop Acoust. Echo and Noise Control, pp. 1-4.

[18] Wang, D. (2008). Time-frequency masking for speech separation and its potential for hearing aid design. Trends in Amplification, 12(4), 332-353. doi: $10.1177 / 1084713808326455$

[19] Wang, D. (2005). On ideal binary mask as the computational goal of auditory scene analysis. In Speech separation by humans and machines, pp: 181-197.doi:10.1007/0-387-22794-6_12

[20] Gray R.M. (2013). Vector Quantization. IEEE ASSP Magazine, 1(2), 4-29.

[21] Likas A., Vlassis and Verbeek J. J., (2003). The global k-means clustering algorithm. Pattern Recognition, 36(2), 451-461.

[22] Khan S. S and Ahmed A. (2004). Cluster center initialization for Kmeans algorithm. Pattern Recognition Letters, 25(11), 1293-1302.

[23] $\mathrm{Hu}$ Y. and Loizou P. (2007). Subjective evaluation and comparison of speech enhancement algorithms. Speech Commun., 49(7-8), 588-601. doi:10.1016/j.specom.2006.12.006 
[24] Rix A.W., Beerends J. G., Hollier M. P., Hekstra A.P. (2001). Perceptual evaluation of speech quality (PESQ)-a new method for speech quality assessment of telephone networks and codecs. In Acoustics, Speech, and Signal Processing (ICASSP), 749-752. doi: 10.1109/ICASSP.2001.941023

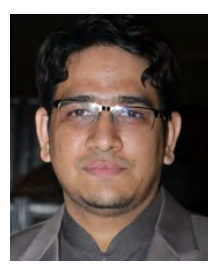

Nasir Saleem

Engr. Nasir Saleem received the B.S degree in Telecommunication Engineering from University of Engineering and Technology, Peshawar-25000, Pakistan in 2008 and M.S degree in Electrical Engineering from CECOS University, Peshawar, Pakistan in 2012. He was a senior Lecturer at the Institute of Engineering and Technology, Gomal University, D.I.Khan-29050, Pakistan. He is now Assistant Professor in Department of Electrical Engineering, Gomal University, Pakistan. Currently, he is pursuing Ph.D. degree in electrical Engineering from University of Engineering and Technology, Peshawar-25000, Pakistan. His research interests are in the area of digital signal processing, speech processing and speech enhancement.

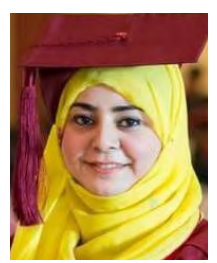

Tayyaba Gul Tareen

Engr. Tayyaba Gul Tareen received the B.S degree in Electrical Engineering from University of Engineering and Technology, Peshawar-25000, Pakistan in 2008 and M.S degree in Electrical Engineering from CECOS University, Peshawar, Pakistan in 2012. Currently, She is pursuing $\mathrm{Ph} . D$. degree in electrical Engineering from Iqra University, Peshawar-25000, Pakistan. Her research interests are in the area of digital signal processing. 